\title{
Gender Dysphoria Disrupting the Course of Treatment of a Recurrent Juvenile Granulosa Cell Tumor in an Adolescent Female: A Case Report
}

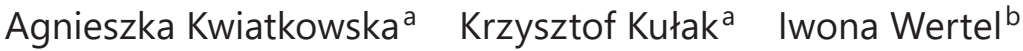 \\ alst Chair and Department of Gynaecologial Oncology and Gynaecology, Medical University \\ of Lublin, Lublin, Poland; ' Independent Laboratory of Cancer Diagnostics and Immunology, \\ Ist Chair and Department of Oncological Gynaecology and Gynaecology, Medical University \\ of Lublin, Lublin, Poland
}

\section{Keywords}

Juvenile granulosa cell tumor - Gender dysphoria - Therapeutic difficulties · Ethical issues

\section{Abstract}

We present the case of an adolescent female patient with gender dysphoria (GD) who was diagnosed with a recurrent ovarian neoplasm - juvenile granulosa cell tumor (JGCT). The 17-year-old female patient presented multiple endocrine pathologies and a recurrent JGCT. During the surgery qualification process, the patient admitted having identified herself as a male. The patient reported being uncomfortable with her body and with the expected roles of her assigned gender. Due to that, the patient requested a total hysterectomy with a bilateral salpingo-oophorectomy. As a minor, she required the permission of her parents, which was not granted. The patient underwent several specialist consultations, after which she agreed to the unilateral removal of tumor-changed pathologies and additional hormonal, psychological, and psychiatric diagnostics. To the best of our knowledge, this is the first detailed report of co-occurrence of GD spectrum disorders and JGCT in an adolescent female. This case contains many therapeutic and ethical problems regarding both physical and mental health. It should be noted that adolescents with GD spectrum rarely develop persistent transsexuality. Modulations from developmental psychology, psychotherapy, family dynamics, hormonal treatment, and the removal of JGCT in the presented case may have potential therapeutic implications for GD. 


\section{Introduction}

The term gender dysphoria (GD) denotes incongruence between the experienced or expressed gender and the sex assigned at birth. Consolidation of gender identity is the main development goal of adolescence. There is still insufficient research about how gender identity and gender variance actually evolve $[1,2]$. In the entire population of children and adolescents, it affects approx. $1 \%$ of the spectrum.

The GD etiology is still unknown [3]. It is suspected that the increased likelihood of GD coexists with increased levels of androgens during pregnancy. Testosterone determines sexual differentiation of the human brain during fetal development [2]. GD is often accompanied by mental disorders such as schizophrenia, borderline personalities, and autism [4]. Neuroanatomical factors affect the occurrence of GD. Experiencing childhood abuse, neglect, and physical or sexual violence may be associated with an increased risk of GD development [2]. However, all of the above-mentioned factors require further clinical validation.

Ovarian granulosa cell tumor (GCT) is a neoplasm that develops from the sex cords and the stroma of the ovary. Juvenile granulosa cell tumor (JGCT) occurs in less than 5\% of GCT cases. Primary ovarian tumors like JGCT in children and adolescent girls are extremely rare and represent less than $1 \%$ of all malignancies in this age group. GCT secretes multiple sex hormones [5]. Most often, they secrete estradiol [6]; however, testosterone and insulin were elevated in the presented case. This form of tumor activity is very rare.

\section{Case Presentation}

A 17-year-old female patient was diagnosed and treated in the Department of Oncological Gynecology and Gynecology due to a suspected recurrence of JGCT. The patient began menstruating at the age of 12 . At the age of 15, menses became irregular and, in addition, hormonal disorders and dermatological problems appeared. The following diagnoses were made after a number of diagnostic tests: hypothyroidism, insulin resistance, psoriasis, and hirsutism. The patient received appropriate treatment and was under constant care of specialist clinics. Before 2018, she did not undergo surgery. In her family, there were no malignancies. In 2018, the patient underwent laparoscopic enucleation of the left ovarian tumor. Following the histopathological result, FIGO (International Federation of Gynecology and Obstetrics) IA folliculoma was diagnosed. The patient was informed of the need to undergo regular physical examination, imaging, and laboratory tests every 3-4 months to evaluate the efficacy of surgical treatment and early detection of a potential relapse. The first two follow-up visits showed no cause for concern. At 9 months, the ultrasound examination showed a tumor in the left ovary, $7 \mathrm{~cm}$ in diameter, suggesting a recurrence of folliculoma. Magnetic resonance imaging confirmed the initial diagnosis (Fig. 1). The patient did not suffer any pain, the laboratory test results were within the normal range, and there were no hormonal fluctuations, except a high level of testosterone and insulin (Table 1). The patient was qualified for surgery. Potential therapeutic options were discussed with her and her parents, paying attention to the patient's young age and the need for fertility preservation. The patient did not agree to the recommended unilateral oophorectomy and staging procedures. She demanded removal of the uterus and adnexa on both sides. During the preoperative workup, the patient confessed having GD. The patient's mother who was present during the conversation confirmed this. For about a year, the patient had had difficulty identifying with her own gender. She was increasingly thinking of undergoing surgery and hormonal treatment to become a male. The patient's mother admitted that her daughter had been mentioning her desire to change sex for some time. The parents talked to the patient about

\section{Karger'}


Fig. 1. Magnetic resonance imaging of the pelvic organs showed a change in the left ovary, $7 \mathrm{~cm}$ in diameter, suggesting a recurrence of folliculoma.

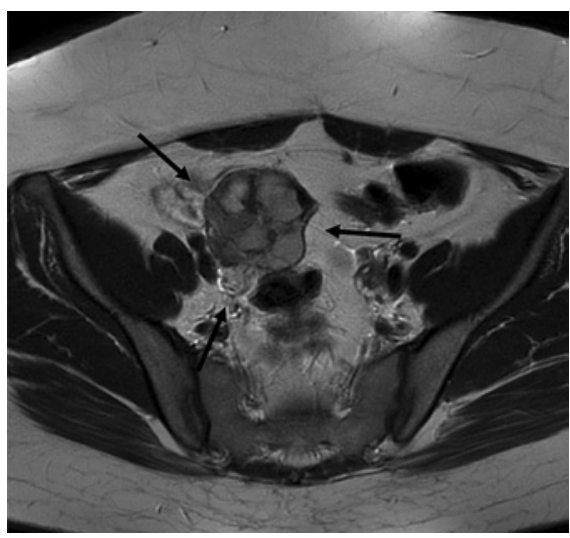

Tab. 1. Laboratory test results

\begin{tabular}{lcc}
\hline Characteristic & Index & Normal range \\
\hline AFP & $3.2 \mathrm{kU} / \mathrm{L}$ & $<6-7 \mathrm{kU} / \mathrm{L}$ \\
E2 & $750 \mathrm{pg} / \mathrm{mL}$ & $11.2-970 \mathrm{pg} / \mathrm{mL}(0.3-3.5 \mathrm{nmol} / \mathrm{L})$ \\
$\mathrm{FSH}$ & $10.7 \mathrm{mIU} / \mathrm{mL}$ & $3-12 \mathrm{mIU} / \mathrm{mL}$ \\
$\mathrm{LH}$ & $12.9 \mathrm{mlU} / \mathrm{mL}$ & $2-13 \mathrm{mIU} / \mathrm{mL}$ \\
$\mathrm{Ca}-125$ & $27.96 \mathrm{U} / \mathrm{mL}$ & $0-35 \mathrm{U} / \mathrm{mL}$ \\
$\mathrm{HE} 4$ & $36.7 \mathrm{pmol} / \mathrm{L}$ & $0-60.5 \mathrm{pmol} / \mathrm{L}$ \\
$\mathrm{hCG}$ & $2 \mathrm{mlU} / \mathrm{mL}$ & $<5 \mathrm{mIU} / \mathrm{mL}$ \\
Testosterone & $14.96 \mathrm{nmol} / \mathrm{L}$ & $0.17-2.81 \mathrm{nmol} / \mathrm{L}$ \\
Insulin & $19.7 \mu \mathrm{J} / \mathrm{mL}$ & $<12 \mu \mathrm{J} / \mathrm{mL}$ \\
\end{tabular}

AFP, $\alpha$-fetoprotein; E2, estradiol; FSH, follicle-stimulating hormone; LH, luteinizing hormone; Ca-125, carcinoma antigen 125; HE4, human epididymis protein 4 ; hCG, human chorionic gonadotropin.

her potential GD, but they did not agree to any medical actions that could affect her appearance and loss of reproductive function. Also they did not consent to a total hysterectomy with bilateral salpingo-oophorectomy. Their decision was associated with the patient's young age and irreversible consequences of the sex change procedure.

Due to the discrepancies in the decision regarding the treatment of the minor, where consent of both the patient and legal guardian are needed, consultations with a psychologist, psychiatrist, and endocrinologist were requested. The consultations concluded in the need of further diagnostics after the oncological treatment. It cannot be excluded that the patient's position is influenced by her hormonal disorders, the presence of the hormonally active ovarian tumor, or other factors: environmental, family, or mental. However, it takes several months to make a correct diagnosis. The diagnosis did not allow such a delay in surgery. After several meetings with the psychologist, psychiatrist, other members of the therapeutic team, and the parents, the patient agreed to unilateral removal of the appendages and staging procedures. Two days after the surgery, the serum total testosterone level declined to 4.87 nmol/L. Postoperative pathology confirmed a unilateral FIGO IA relapse of the JGCT. The patient did not decide to undergo the proposed adjuvant chemotherapy, which was recommended and scheduled due to the recurrent nature of the disease. The patient is currently undergoing diagnostics for GD and considering various therapeutic options. She is prone to maintaining fertility by the oocyte cryptopreservation method before sex change. 
Kwiatkowska et al.: GD Disrupting the Course of Treatment of a Recurrent JGCT

\section{Discussion/Conclusion}

Doctors need to give the best care for the patient's health, according to the Hippocratic Oath and the principles of medical ethics. This should also be understood as challenges associated with the therapeutic process, as in this case [7]. GD patients require a multidisciplinary approach [1].

Most juvenile GCTs secrete estradiol [5,6]. Only several cases of JGCT secreting androgens causing virilization have been reported. This is confirmed by the present case, as the patient had hirsutism, elevated testosterone, and abdominal obesity, which is more common in men [6]. This clinical picture may suggest a potential effect on the occurrence of GD. It has been confirmed that increased levels of androgens modulate the onset of GD [8]. In addition, the presence of hirsutism could additionally affect the patient's disturbed image of her sexuality.

In the present case, the histopathological examination revealed granular cells with central nuclear atypia and common mitotic activities, and large eosinophilic cytoplasm as well as cells with an irregular size, follicular structures, and absence of Call-Exner bodies, which is characteristic of JGCT. The immunohistochemical evaluation showed positive results for $\alpha$-inhibin, CD 99, and calretinin, which are currently the most specific parameters in the diagnosis of JGCT $[5,6]$. In the described case, none of the above-mentioned etiological factors of GD were confirmed.

The guidelines of the Diagnostic and Statistical Manual of Mental Disorders, Fifth Edition (DSM-5) specify a period of at least 6 months of the presence of symptoms required before GD diagnosis. Our patient met the criteria, as the symptoms had persisted for 1 year. She also presents a clear discrepancy between the experienced gender and the assigned female gender, which is manifested in a strong desire to be rid of primary sex characteristics due to the clear incompatibility with the experienced sex [2]. However, this is not sufficient to make a GD diagnosis [2, 4]. Detailed examinations for GD should be conducted by mental health professionals [9] who have sufficient training and experience in assessing related mental health conditions and are willing to participate in the ongoing care throughout the endocrine transition $[1,2,9]$. In the case of our patient, the endocrinologist and oncologist-gynecologist should be included in the diagnostic process due to the hormonal disorders and recurrent ovarian tumor $[3,9]$.

The patient is still in puberty. Her hormonal balance is dysregulated, which can be seen in the presence of concomitant disorders and clinical symptoms. In addition, she has been diagnosed with a hormone-active ovarian tumor, which can also affect hormonal fluctuations. The review of Solomon and Conner presents an insight of endocrine diseases manifesting itself in psychiatric disorders [2]. Available literature shows that GD female-to-men hormone treatment relies on the supply of testosterone $[9,10]$. Our patient had significantly increased testosterone levels without supplementation before the surgery, which immediately decreased after the surgery. This can be considered as an additional factor determining the presence of the GD and the strong desire to remove the uterus and appendages.

Another possible diagnosis to consider is the initial phase of borderline personality disorder, mood disorder, dissociative disorder, psychotic disorder, and schizophrenia. It can also be simply a manifestation of the "period of youth rebellion" or a desire for parents' attention. Schizophrenia and borderline personality are diagnosed at a young age, so it is potentially possible in this case. In addition, even for a longer time, it may be accompanied by delusions, for example, of one's own sex [2, 4].

In the case described in this report, there are many ethical issues. According to Polish law, a minor patient over the age of 16 requires double consent (combined consent) for an operation, treatment method, or invasive diagnostics procedures. Medical history and gynecological examination must be carried out in the presence of the parent after written consent

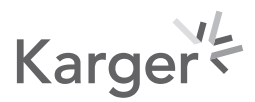


[11]. Dissonance between the expectations of the patient and her legal guardians appeared during the surgical workup. The patient was expecting radical surgery, whereas the parents insisted on conserving surgery. Under Polish law, it is not possible to perform surgery for which one side agrees and the other does not.

It should be noted that, in the case of recurrent JGCT, total abdominal hysterectomy and bilateral salpingo-oophorectomy is the recommended method [12, 13]. However, in the case of such a young patient, gynecologists should also take care of her reproductive health $[1,7]$. Even the slightest chance to avoid such radical surgery should be considered by experts and applied if possible, as in the case of this patient. Specialists in gynecology and oncology concluded that the best method in this case would be unilateral salpingooophorectomy with staging procedures $[5,13]$. In addition, adjuvant chemotherapy should be used after surgery.

Several consultations with various specialists were also provided for the patient and her parents to make a clear decision regarding the surgery. It was important because JGCT is a fast-growing tumor. The situation was complicated by the fact that it was a recurrence. The patient insisted on her arguments, and the parents wanted her to choose, but only after coming of age. After a fertility sparing operation, she may undergo surgical sex change procedures in the future and, additionally, fertility preservation procedures $[5,7,14]$. There would be no such possibility after radical surgery. The impulsive nature of adolescents also prohibits foresight. The desire for procreation may appear later in the patient's life, regardless of her current sex status. Radical surgery is irreversible, and the uterine transplant is still a pioneer and scarcely available method. It is also worth mentioning here that, as shown by the available literature, a large proportion of adolescents with the GD spectrum do not develop a permanent form of transsexualism $[1,2]$. Gynecologists must take special care of the reproductive health of patients [15], even if the latter under care for oncological reasons do not consider having offspring $[1,14]$. In addition, the patient was informed that hysterectomy and bilateral oophorectomy was associated with many side effects and risks.

There are diverse contraceptive options and each patient can choose the perfect one without interfering with reproductive organs. Skordis et al. [2] point out that solutions for maintaining fertility in adolescent children and adolescents are needed. In a German study, Auer et al. [14] have noted that a large percentage of transgender patients wish to have children, but unfortunately the prevention of fertility during sex change procedures is low.

Due to the early recurrence of JGCT in the patient, it is necessary to continue thorough follow-ups that will be able to capture the next recurrence of JGCT as soon as possible. The most prudent decision would be to postpone the start of the sex change process for 5 years after surgery [13]. This is dictated by the patient's health and oncological vigilance.

According to the guidelines, hormone therapy with testosterone is contraindicated in patients with previous cancer history. This applies to active tumors that are sensitive to sex hormones. The use of hormone therapy in this patient, which is usually the first phase of sex change, is uncertain. If the therapeutic team consensus allows gender-affirming hormone therapy, the dosing should be carefully established [9]. Cross-sex hormones with or without progestins/GnRH agonists introduction should be considered with reference to the patient's age in accordance with the recommendations and guidelines of World Professional Association for Transgender Health (WPATH)/Endocrine Society Clinical Guideline regarding the treatment of GD $[3,9,10]$.

The WPATH proposes delaying genital gender reassignment surgery, with the exception of chest surgery in trans men, which may be performed earlier. The clinician responsible for care during hormonal transformation must confirm that surgery is necessary and will be beneficial for the overall patient's health and well-being. If there are contraindications to the use of hormone therapy, the procedure may be considered before this time $[3,10]$.

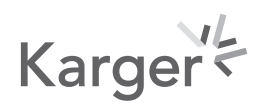


To the best of our knowledge, this is the first detailed case report describing the presence of JCGT and coexistence of GD spectrum disorders. It has been shown that a hormonally active tumor may have severe implications for GD. There are many therapeutic and ethical problems regarding both physical and mental health that have to be faced by a physician team in this case. It is necessary to ensure the possibilities of maintaining fertility and mental health in the young woman especially during oncological treatment. The case shows that empathy and understanding of patients' issues has measurable benefits. Multidisciplinary cooperation of mental health professionals, endocrinologists, and oncological gynecologists also plays an important role in clinical management.

\section{Acknowledgement}

We extend our sincere appreciation to the patient's family for their support.

\section{Statement of Ethics}

Written ethical approval was obtained from the patient and her parents for the publication of this case report.

\section{Conflict of Interest Statement}

The authors have no conflicts of interest to declare.

\section{Funding Sources}

This paper did not receive any specific grant from funding agencies in the public, commercial, or not-for-profit sectors.

\section{Author Contributions}

All authors were involved in the preparation of the manuscript. A.K.: Substantial contributions to the conception or design of the work; or the acquisition, analysis, or interpretation of data for the work; drafting the work or revising it critically for important intellectual content. K.K.: Lead physician responsible for patient care, performed the ultrasound and the surgery; revising the work critically for important intellectual content. I.W.: Revising the work it critically for important intellectual content and approved the final manuscript as submitted. All authors approved the final manuscript as submitted and agree to be accountable for all aspects of the work.

\section{Karger'}




\section{References}

1 Mehringer J, Dowshen NL. Sexual and reproductive health considerations among transgender and genderexpansive youth. Curr Probl Pediatr Adolesc Health Care. 2019;49(9):100684.

2 Skordis N, Kyriakou A, Dror S, Mushailov A, Nicolaides NC. Gender dysphoria in children and adolescents: an overview. Hormones (Athens). 2020 Sep;19(3):267-76.

3 Coleman E, Bockting W, Botzer M, Cohen-Kettenis P, DeCuypere G, Feldman J, et al. Standards of care for the health of transsexual, transgender and gender non-conforming people, version 7. Int J Transgenderism. 2011; 13:165-232.

4 Urban M. [Transsexualism or delusions of sex change? Avoiding misdiagnosis]. Psychiatr Pol. 2009;43(6): 719-28.

5 Zhao D, Song Y, Zhang Y, Li B. Outcomes of Fertility-Sparing Surgery in Ovarian Juvenile Granulosa Cell Tumor. Int J Gynecol Cancer. 2019;29(4):787-91.

6 Young RH, Dickersin GR, Scully RE. Juvenile granulosa cell tumor of the ovary. A clinicopathological analysis of 125 cases. Am J Surg Pathol. 1984;8(8):575-96.

7 Bizic MR, Jeftovic M, Pusica S, Stojanovic B, Duisin D, Vujovic S, et al. Gender Dysphoria: Bioethical Aspects of Medical Treatment. Biomed Res Int. 2018;2018:9652305.

8 Solomon SS, Conner SH. Psychiatric Manifestations of Endocrine Disorders. J Hum Endocrinol. 2017;2:007.

9 Hembree WC, Cohen-Kettenis PT, Gooren L, Hannema SE, Meyer WJ, Murad MH, et al. Endocrine Treatment of Gender-Dysphoric/Gender-Incongruent Persons: An Endocrine Society Clinical Practice Guideline. J Clin Endocrinol Metab. 2017;102(11):3869-903.

10 Mahfouda S, Moore JK, Siafarikas A, Hewitt T, Ganti U, Lin A, et al. Gender-affirming Hormones and Surgery in Transgender Children and Adolescents. Lancet Diabetes Endocrinol. 2019;7(6):484-98.

11 Ustawa z dnia 6 listopada 2008 r. o prawach pacjenta i Rzeczniku Praw Pacjenta. Dz. U. 2009;52:417.

12 Ray-Coquard I, Morice P, Lorusso D, Prat J, Oaknin A, Pautier P, et al. Non-epithelial ovarian cancer: ESMO Clinical Practice Guidelines for diagnosis, treatment and follow-up. Ann Oncol. 2018;29(Suppl 4):iv1-iv18.

13 Basta A, Bidziński M, Bieńkiewicz A, Blecharz P, Bodnar L, Jach R, et al. Recommendations of the Polish Gynecological Oncology Society for the diagnosis and treatment of ovarian cancer. Curr Gynecol Oncol. 2017;15(1): 5-23.

14 Auer MK, Fuss J, Nieder TO, Briken P, Biedermann SV, Stalla GK, et al. Desire to Have Children Among Transgender People in Germany: A Cross-Sectional Multi-Center Study. J Sex Med. 2018;15(5):757-67.

15 Unger CA. Care of the transgender patient: the role of the gynecologist. Am J Obstet Gynecol. 2014;210(1): $16-26$. 